EPJ Web of Conferences 81, 01003 (2014)

DOI: $10.1051 /$ epjconf/ 20148101003

(C) Owned by the authors, published by EDP Sciences, 2014

\title{
HADES results in elementary reactions
}

\section{B.Ramstein ${ }^{1, a}$ for the HADES Collaboration}

J. Adamczewski-Musch ${ }^{2}$, O. Arnold ${ }^{3,4}$, E.T. Atomssa1 ${ }^{1}$, C. Behnke ${ }^{5}$, J.C. Berger-Chen ${ }^{3,4}$, J. Biernat ${ }^{6}$, A. Blanco ${ }^{7}$, C. Blume ${ }^{5}$, M. Böhmer ${ }^{3}$, P. Bordalo $^{7}$, S. Chernenko ${ }^{8}$, C. Deveaux ${ }^{9}$, A. Dybczak ${ }^{6}$, E. Epple ${ }^{3,4}$, L. Fabbietti ${ }^{3,4}$, O. Fateev ${ }^{8}$, P. Fonte ${ }^{7, b}$, C. Franco ${ }^{7}$, J. Friese ${ }^{3}$, I. Fröhlich ${ }^{5}$, T. Galatyuk $^{10, c}$, J. A. Garzón ${ }^{11}$, K. Gill ${ }^{5}$, M. Golubeva ${ }^{12}$, F. Guber ${ }^{12}$, M. Gumberidze ${ }^{10, c}$, S. Harabasz ${ }^{6,10}$, T. Hennino ${ }^{1}$, S. Hlavac ${ }^{13}$, C. Höhne ${ }^{9}$, R. Holzmann ${ }^{2}$, A. lerusalimov ${ }^{8}$, A. Ivashkin ${ }^{12}$, M. Jurkovic ${ }^{3}$, B. Kämpfer ${ }^{14, d}$, T. Karavicheva ${ }^{12}$, K. Kardan ${ }^{5}$, I. Koenig ${ }^{2}$, W. Koenig ${ }^{2}$, B. W. Kolb ${ }^{2}$, G. Korcyl ${ }^{6}$, G. Kornakov ${ }^{10}$, R. Kotte ${ }^{14}$, A. Krása ${ }^{15}$, E. Krebs ${ }^{5}$, H. Kuc ${ }^{6,1}$, A. Kugler ${ }^{15}$, T. Kunz ${ }^{3}$, A. Kurepin ${ }^{12}$, A. Kurilkin ${ }^{8}$, P. Kurilkin ${ }^{8}$, V. Ladygin ${ }^{8}$, R. Lalik ${ }^{3,4}$, K. Lapidus ${ }^{3,4}$, A. Lebedev ${ }^{16}$, L. Lopes ${ }^{7}$, M. Lorenz ${ }^{5}$, T. Mahmoud ${ }^{9}$, L. Maier ${ }^{3}$, A. Mangiarotti ${ }^{7}$, J. Markert $^{5}$, V. Metag ${ }^{9}$, J. Michel ${ }^{5}$, C. Müntz ${ }^{5}$, R. Münzer ${ }^{3,4}$, L. Naumann ${ }^{14}$, M. Palka ${ }^{6}$, Y. Parpottas ${ }^{17, e}$, V. Pechenov ${ }^{2}$, O. Pechenova ${ }^{5}$, V. Petousis ${ }^{17}$, J. Pietraszko ${ }^{2}$, W. Przygoda ${ }^{6}$, L. Rehnisch ${ }^{5}$, A. Reshetin ${ }^{12}$, A. Rost $^{10}$, A. Rustamov ${ }^{5}$, A. Sadovsky ${ }^{12}$, P. Salabura ${ }^{6}$, T. Scheib ${ }^{5}$, K. SchmidtSommerfeld $^{3}$, H. Schuldes ${ }^{5}$, P. Sellheim ${ }^{5}$, J. Siebenson ${ }^{3}$, L. Silva ${ }^{7}$, Yu.G. Sobolev ${ }^{16}$, S. Spataro ${ }^{\dagger}$, H. Ströbele ${ }^{5}$, J. Stroth ${ }^{5,2}$, P. Strzempek ${ }^{6}$, C. Sturm ${ }^{2}$, O. Svoboda ${ }^{15}$, A. Tarantola ${ }^{5}$, K. Teilab ${ }^{5}$, P. Tlusty ${ }^{15}$, M. Traxler ${ }^{2}$, H. Tsertos ${ }^{17}$, T. Vasiliev ${ }^{8}$, V. Wagner ${ }^{15}$, C. Wendisch ${ }^{14, d}$, J. Wirth ${ }^{3,4}$, J. Wüstenfeld ${ }^{14}$, Y. Zanevsky ${ }^{8}$, and P. Zumbruch ${ }^{2}$

\footnotetext{
${ }^{1}$ Institut de Physique Nucléaire (UMR 8608), CNRS/IN2P3 - Université Paris Sud, F-91406 Orsay Cedex, France

${ }^{2}$ GSI Helmholtzzentrum für Schwerionenforschung GmbH, 64291 Darmstadt, Germany

${ }^{3}$ Physik Department E12, Technische Universität München, 85748 Garching, Germany

${ }^{4}$ Excellence Cluster 'Origin and Structure of the Universe', 85748 Garching, Germany

${ }^{5}$ Institut für Kernphysik, Goethe-Universität, 60438 Frankfurt, Germany

${ }^{6}$ Smoluchowski Institute of Physics, Jagiellonian University of Cracow, 30-059 Kraków, Poland

${ }^{7}$ LIP-Laboratório de Instrumentação e Física Experimental de Partículas , 3004-516 Coimbra, Portugal

${ }^{8}$ Joint Institute of Nuclear Research, 141980 Dubna, Russia

${ }^{9}$ II.Physikalisches Institut, Justus Liebig Universität Giessen, 35392 Giessen, Germany

${ }^{10}$ Technische Universität Darmstadt, 64289 Darmstadt, Germany

${ }^{11}$ LabCAF. F. Física, Univ. de Santiago de Compostela, 15706 Santiago de Compostela, Spain

${ }^{12}$ Institute for Nuclear Research, Russian Academy of Science, 117312 Moscow, Russia

${ }^{13}$ Institute of Physics, Slovak Academy of Sciences, 84228 Bratislava, Slovakia

${ }^{14}$ Institut für Strahlenphysik, Helmholtz-Zentrum Dresden-Rossendorf, 01314 Dresden, Germany

${ }^{15}$ Nuclear Physics Institute, Academy of Sciences of Czech Republic, 25068 Rez, Czech Republic

${ }^{16}$ Institute of Theoretical and Experimental Physics, 117218 Moscow, Russia

${ }^{17}$ Department of Physics, University of Cyprus, 1678 Nicosia, Cyprus

${ }^{b}$ also at ISEC Coimbra, Coimbra, Portugal

${ }^{c}$ also at ExtreMe Matter Institute EMMI, 64291 Darmstadt, Germany

${ }^{d}$ also at Technische Universität Dresden, 01062 Dresden, Germany

${ }^{e}$ also at Frederick University, 1036 Nicosia, Cyprus
}

\footnotetext{
ae-mail: ramstein@ipno.in2p3.fr
} 


\begin{abstract}
Recent results obtained with the HADES experimental set-up at GSI are presented with a focus on dielectron production and strangeness in $p p$ and quasi-free $n p$ reactions. Perspectives related to the very recent experiment using the pion beam at GSI are also discussed.
\end{abstract}

\title{
1 Introduction
}

The main goal of the heavy-ion collision experiments with the High Acceptance Di-Electron spectrometer (HADES) at GSI is the study of hadronic matter in the 1-3.5 GeV/nucleon incident energy range where moderate temperatures $(T<80 \mathrm{MeV}$ ) and large densities (up to 3 times the normal nuclear matter density) are reached. Rare penetrating probes, like dielectrons or strange particles, are used for such studies. The present interpretation of dilepton spectra which are measured by several experiments, mainly operating at ultra-relativistic energies, is based on hadronic models, which predict in-medium modifications of the $\rho$ meson spectral function due to their coupling to resonance-hole states [1]. In the energy range of the HADES experiments, the $\rho$ meson is mainly produced in primary NN or secondary $\pi \mathrm{N}$ collisions where the coupling of the $\rho$ meson to baryonic resonances plays already a role. This opens the possibility to study these effects and therefore constrain the interpretation of medium effects by measuring dielectron emission in elementary reactions. An additional interest of studying the couplings of $\rho$ meson to the baryonic resonances is the close connection to the electromagnetic structure of the baryonic transitions, which is explicit, in particular in the Vector Dominance Models (VDM). Strange particles can also be used to investigate medium effects. For example the strength of the kaon in-medium potential is still an open question and has connection with the equation of state of the nuclear matter. The production mechanisms of strange particles are also poorly known, especially when they are produced far below threshold. Elementary reactions can again provide very important constraints, for example concerning the role of baryonic resonances in the production mechanisms. The strength of kaon-nucleon potential can also be investigated, by studying the production of kaons, of $\Lambda(1405)$ or by looking for a signal of $K^{-} p p$ bound states. Based on these motivations, in addition to the study of hot and dense nuclear matter (studied in nucleus-nucleus collisions) and cold nuclear matter (studied in $p+A$ or $\pi+A$ reactions), the HADES experimental program, which is the main topics of this talk, comprises measurements in $p p$, "quasifree" $n p$ or $\pi^{-}+p$ reactions.

\section{Dielectron emission}

The spectrometer HADES [2] allows for the detection of $e^{+} e^{-}$pairs and charged hadrons in full azimuth and in the polar angle range between $18^{\circ}$ and $85^{\circ}$. In addition, a scintillator wall placed $7 \mathrm{~m}$ downstream the HADES target was used to detect spectator protons in the experiments using a deuterium beam in order to study the quasi-free $n p$ reaction. For the future experiments at FAIR, an electromagnetic calorimeter will be installed [3]. The pp reaction was measured at three incident beam energies, $1.25 \mathrm{GeV}, 2.2 \mathrm{GeV}$ and $3.5 \mathrm{GeV}$. At the lowest energy, which is below the $\eta$ production threshold, the dielectron production has two main origins: the $\pi^{0}$ Dalitz decay $\left(\pi^{0} \rightarrow \gamma e^{+} e^{-}\right)$, which contributes at $e^{+} e^{-}$invariant masses below the $\pi^{0}$ mass, and the $\Delta$ Dalitz decay $\left(\Delta \rightarrow N e^{+} e^{-}\right)$, which extends up to the kinematical limit. The non-resonant pp Bremstrahlung contribution is indeed expected to have a small contribution. The dominance of the $\Delta$ Dalitz decay process above the $\pi^{0}$ 
mass was confirmed by the analysis of the exclusive channel $p p \rightarrow p p e^{+} e^{-}$. A first measurement of the branching ratio of this process could be extracted, and was found consistent with the QED calculation, taking into account the values of the electromagnetic form factor at the photon point (i.e. $G_{M}=3$, $G_{E}=0, G_{C}=0$ ). This study was supported by the measurement of the one-pion production channels, which are simultaneously measured by HADES [4]. A Partial Wave Analysis (PWA) of these onepion channels is also on-going to constrain even more precisely the size of the $\Delta$ contribution, as presented in this conference [5].
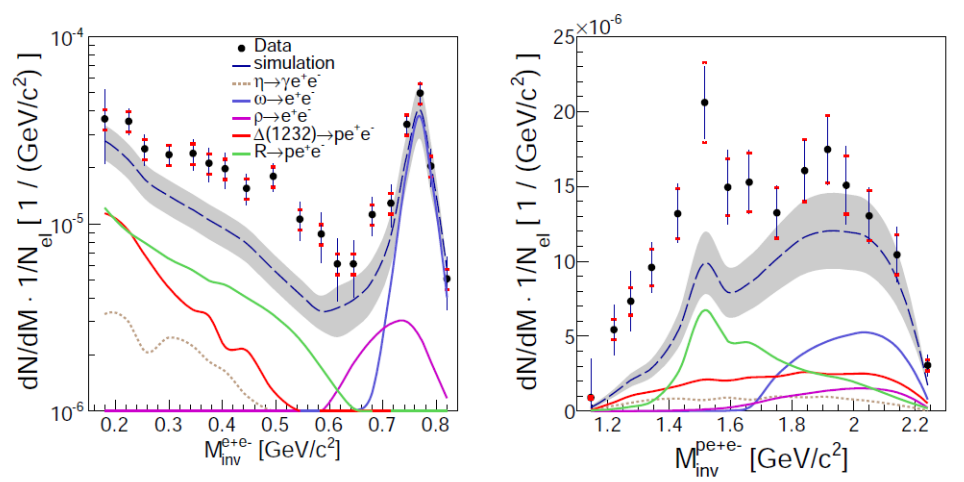

Figure 1. Picture from [6]. $e^{+} e^{-}$(left) and $p e^{+} e^{-}$(right) invariant mass distributions measured in the $p p \rightarrow p p e^{+} e^{-}$reaction at 3.5 $\mathrm{GeV}$ are compared to the simulation result assuming a point-like $R N \gamma^{\star}$ coupling. The hatched area indicates the model errors.

At 2.2 and $3.5 \mathrm{GeV}$, the interpretation of the inclusive spectra $[7,8]$ has to include the $\eta$ Dalitz decay contribution and the $\omega$ and $\rho$ direct decays as well. A detailed study was performed at $3.5 \mathrm{GeV}$ by combining the exclusive one pion production $\left(p p \rightarrow p p \pi^{0}\right.$ and $\left.p p \rightarrow p n \pi^{+}\right)$and dielectron $(p p \rightarrow$ $p p e^{+} e^{-}$) channels [6]. The invariant masses and angular distributions of the pionic channels were first analyzed to fix the contributions of the different baryonic resonances. Their known couplings to $\gamma \mathrm{N}$, which are related to their radiative decays, were then used to calculate the dilepton yield corresponding to their Dalitz decay. After adding the contribution of the $\rho$ and $\omega$ direct decays, this cocktail was compared to the dielectron invariant mass measured in the $p p \rightarrow p p e^{+} e^{-}$channel. As shown in the left part of figure 1, this cocktail underestimates the dielectron yield in the region below the vector meson poles. This effect is due to the electromagnetic form factors of the baryonic transitions, or in the hadron-meson language to the coupling of the $\rho$ meson to the baryonic resonances, which were not included. In addition, it could be deduced from the invariant mass of the $p e^{+} e^{-}$system that the effect is mainly due to the lightest baryonic resonances, and most probably to the $N(1520)$ (right part of figure 1). More precisely, the production of the $\rho$ meson via light baryonic resonances $(N(1520, N(1535), \ldots)$ favors the production of mesons with a low mass, which reflects in a higher yield at lower $e^{+} e^{-}$invariant masses. This distortion of the $\rho$ meson spectral function in $N N$ collision has the same origin as the in-medium one. Therefore, these data offer a way to check the interpretation of in-medium data and in first place the results obtained by the HADES collaboration in the reaction $\mathrm{p}+\mathrm{Nb}$ at $3.5 \mathrm{GeV}[9,10]$.

Large isospin effects could be observed by studying the quasi-free $p n$ reaction using a deuterium beam. The production of dielectron at invariant masses larger than $300 \mathrm{MeV} / \mathrm{c}^{2}$ exceeded by factors up to 10 the predictions based on One-Boson Exchange models (OBE). The latter include non-resonant graphs, which have a stronger effect in $p n$ than in $p p$ collisions. Recently, there were several rather successful attempts to reduce this discrepancy. A VDM pion electromagnetic form factor was included in the calculation of charged current graphs in the OBE model of [11]. In [12], the $n p \rightarrow d e^{+} e^{-}$ contribution was shown to contribute significantly at large $e^{+} e^{-}$invariant masses. The cross-section of this process should be soon extracted from HADES exclusive data. Very recently, an interpretation 
based on virtual $\rho$ production in double $\Delta$ final state interaction was also put forward. In order to constrain this process, the double pion production is analyzed in different channels of the $p p$ and $n p$ reactions $\left(p p \rightarrow p p \pi^{+} \pi^{-}, n p \rightarrow n p \pi^{+} \pi^{-}, n p \rightarrow p p \pi^{0} \pi^{-}, n p \rightarrow d \pi^{+} \pi^{-}\right.$), as discussed in this conference [13]. In addition, these analyses should provide independent checks on the existence of the $d^{\star}$ resonance $\left(M=2.38 \mathrm{GeV} / \mathrm{c}^{2}\right)$ which seemed to be observed by the WASA collaboration (see the contribution [14] at this conference) and could also contribute to the dilepton production, exclusively in $p n$ reactions.

Furthermore, as also presented in detail in this conference [15], the measurement of high statistics dielectron invariant mass spectra with a good resolution made it possible for HADES to improve the upper limit on the kinetic mixing parameter of the dark photon in a range of mass from 0.02 to 0.1 $\mathrm{GeV} / \mathrm{c}^{2}[16]$. The upper limits on the $\eta \rightarrow \gamma \gamma$ branching ratio could also be improved twice in the last years by the HADES collaboration $[8,16]$.

\section{Strangeness production}
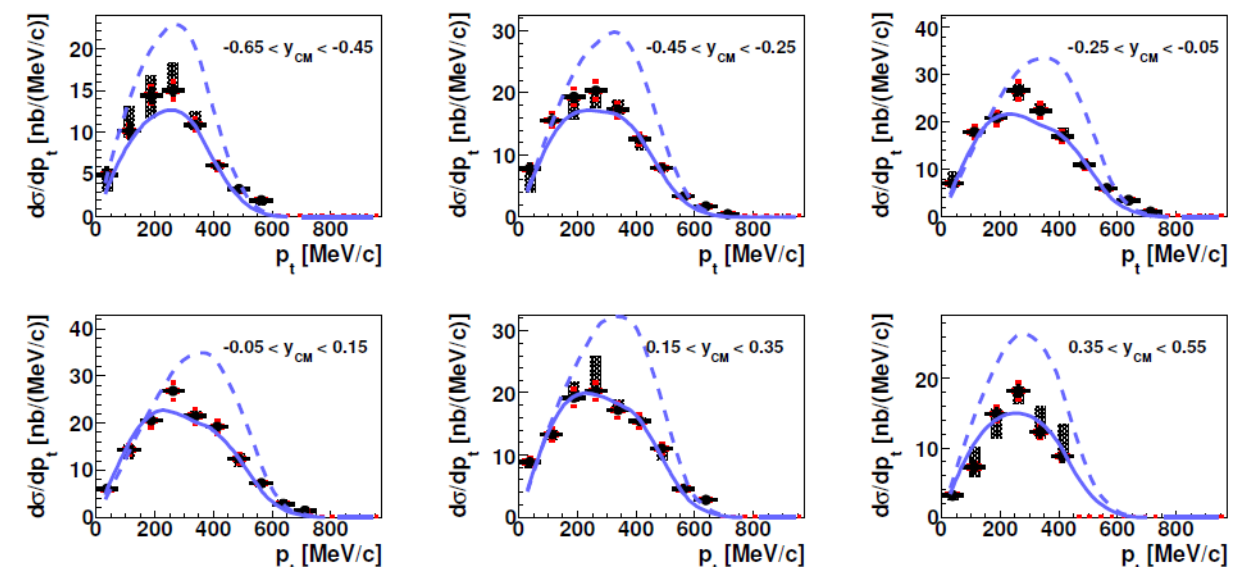

Figure 2. Picture from [17]. Transverse momentum spectra of $K_{S}^{0}$ produced in $\mathrm{p}+\mathrm{p}$ collisions at $3.5 \mathrm{GeV}$. Experimental data (black circles) and GiBUU transport model simulations (dashed curves: original resonance model [18], solid curves: modified resonance model, based on exclusive channel measurements [19]). Systematic uncertainties due to the efficiency correction and absolute normalization are indicated by shaded boxes and red horizontal dashes, respectively.

Strangeness production is also a fruitful part of the HADES program in elementary collisions. As an example, a detailed study of exclusive $K_{S}^{0}$ production in the $p p \rightarrow \Lambda p \pi^{+} K^{0}$ and $p p \rightarrow \Sigma^{0} p \pi^{+} K^{0}$ channels was just published [19], showing the dominant role played by the $\Delta$ resonance in these processes. These results were used to improve existing resonance model predictions for inclusive $K_{S}^{0}$ production which serve in turn as a reference for the in-medium kaon potential studies in the $p+N b$ reaction at $3.5 \mathrm{GeV}$ [17]. A higher sensitivity is expected in the data measured for the $\mathrm{Au}+\mathrm{Au}$ reaction at $1.25 \mathrm{GeV}$, which are currently under analysis. HADES also contributed to the study of the $\Lambda$ (1405) [20]. The two charged decay channels $\Lambda(1405) \rightarrow \Sigma^{ \pm} \pi^{\mp}$ have been reconstructed for the first time in $p p$ collisions and a peak position clearly below $1400 \mathrm{MeV} / \mathrm{c}^{2}$ was measured. The fact that the $\Sigma \pi$ spectra connected to the $\Lambda(1405)$ resonance strongly differ among different reactions points 
MESON 2014 $-13^{\text {th }}$ International Workshop on Production, Properties and Interaction of Mesons

to the different production mechanisms and/or to interference effects between the different channels contributing to the observed final state [21]. Recently, a PWA of the $p p \rightarrow p p K^{-}+K^{+}$was also performed, in order to put upper limits on the cross section for the production of antikaonic nuclear clusters $p p K^{-}$. The results have been presented at this conference [22].

\section{Pion beam experiments}

The pion beam at GSI covers the energy range of the second and third resonance region. It is presently unique in the world and offers the possibility to improve the very scarce data base for pion-nucleon reactions, which is needed for modern PWA or to test Coupled-Channel models [23]. The precise determination of the coupling of the $N(1520)$ to the $\rho$ meson is one of the open questions, which is directly related to the importance of in-medium distortions of the $\rho$ meson spectral function, as discussed above. The measurement of dielectron production in the vicinity of the $N(1520)$ resonance pole can provide a measurement of the Dalitz decay branching ratio of this resonance, which is both related to its electromagnetic structure and to its coupling to the $\rho$ meson vector and for which predictions span over one order of magnitude [24-26]. Finally, the reaction $\pi^{-}+A$ allows to study cold nuclear effects on dielectron and strangeness production. To reconstruct the pion tracks and momentum event-by-event, and select interactions with the target material, in-beam detectors were installed. The experiment was performed this summer, with a restricted program, due to the limited available beam time at GSI, but the main objectives mentioned above should be reached.

\section{Conclusion}

The HADES program in elementary reactions is an indispensable complement to medium effects studies. An overlook of the large variety of results obtained so far was given in this talk, with an emphasis on dilepton and strangeness production. The very recent measurements using the GSI pion beam offer interesting new perspectives, related to the $N(1520) N \rho$ coupling and cold nuclear matter effects on strangeness production. The HADES experimental program will then be continued using the proton and ion beams at FAIR.

\section{Acknowledgements}

The HADES collaboration gratefully acknowledges the support by the grants PTDC/FIS/113339/2009 LIP Coimbra, NCN grant 2013/10/M/ST2/00042 SIP JUC Cracow, Helmholtz Alliance HA216/EMMI GSI Darmstadt, VH-NG-823, Helmholtz Alliance HA216/EMMI TU Darmstadt, 283286, 05P12CRGHE HZDR Dresden, Helmholtz Alliance HA216/EMMI, HIC for FAIR (LOEWE), GSI F\&E Goethe-University, Frankfurt VHNG-330, BMBF 06MT7180 TU München, Garching BMBF:05P12RGGHM JLU Giessen, Giessen UCY/341123100, University Cyprus CNRS/IN2P3, CNRS/IN2P3 (France), MSMT LG 12007, AS CR M100481202, GACR 13-06759S NPI AS CR, Rez, EU Contract No. HP3-283286.

\section{References}

[1] R. Rapp, J. Wambach, Eur. Phys. J. A6, 415 (1999)

[2] G. Agakichiev et al. (HADES collaboration), Eur. Phys. J. A41, 243 (2009)

[3] P. Rodriguez-Ramos et al. (HADES collaboration), contribution to these proceedings

[4] G. Agakishiev et al. (HADES Collaboration), Eur. Phys. J. A48, 74 (2012) 
[5] W. Przygoda et al. (HADES collaboration), contribution to these proceedings

[6] G. Agakishiev, A. Balanda, D. Belver, A. Belyaev, J. Berger-Chen et al., Eur.Phys.J. A50, 82 (2014)

[7] G. Agakishiev et al. (HADES Collaboration), Phys. Rev. C85, 054005 (2012)

[8] G. Agakishiev et al. (HADES Collaboration), Eur. Phys. J. A48, 64 (2012)

[9] G. Agakishiev et al. (HADES COllaboration), Phys. Lett. B715, 304 (2012)

[10] J. Weil, H. van Hees, U. Mosel, Eur. Phys. J. A48, 111 (2012)

[11] R. Shyam, U. Mosel, Phys. Rev. C82, 062201 (2010)

[12] B. Martemyanov, M. Krivoruchenko, A. Faessler, Phys. Rev. C84, 047601 (2011)

[13] A. Kurilkin et al. (HADES collaboration), contribution to these proceedings

[14] H. Clement, contribution to these proceedings

[15] M. Gumberidze et al. (HADES collaboration), contribution to these proceedings

[16] G. Agakishiev et al. (HADES collaboration), Phys. Lett. B731, 265 (2014)

[17] G. Agakishiev et al. (HADES Collaboration), nucl-ex/1404.7011 (2014)

[18] K. Tsushima, A. Sibirtsev, A.W. Thomas, G. Li, Phys. Rev. C59, 369 (1999)

[19] G. Agakishiev et al. (HADES Collaboration), Phys. Rev. C 90, 015202 (2014)

[20] G. Agakishiev et al. (HADES Collaboration), Phys. Rev. C87, 025201 (2013)

[21] J. Siebenson, L. Fabbietti, Phys. Rev. C88, 055201 (2013)

[22] E. Epple et al. (HADES collaboration), contribution to these proceedings

[23] V. Shklyar, H. Lenske, U. Mosel, nucl-th/1409.7920 (2014)

[24] A.I. Titov, B. Kämpfer, Eur. Phys. J. A12, 217 (2001)

[25] M. Lutz, G. Wolf, B. Friman, Nucl. Phys. A706, 431 (2002)

[26] M. Zetenyi, G. Wolf, Phys. Rev. C86, 065209 (2012) 